\title{
Modeling Virologic Response in HIV-1 Infected Patients to Assess Medication Adherence
}

\author{
Yangxin Huang \\ Department of Epidemiology and Biostatistics, College of Public Health, \\ University of South Florida, Tampa, FL,
}

USA

\section{Introduction}

Although the advent of highly active antiretroviral therapy (HAART), including potent protease inhibitors (PIs), has profoundly reduced human immunodeficiency virus (HIV) mortality and morbidity (Palella et al., 1998; CDC, 2009), these combination regimens are not a cure for HIV infection and therapy may be life long. While many patients benefit from HAART treatment, others do not benefit or only experience a temporary benefit. There are several reasons why treatment fails, with poor patient adherence to HAART a leading contributing factor (Ickovics \& Meisler, 1997; Paterson, 2000). Thus, assessment of medication adherence within AIDS clinical trials is a critical component of the successful evaluation of therapy outcomes. Maintaining adherence may be particularly difficult when the drug regimen is complex or side-effects are common, as is often the case for current HIV therapy especially in highly treatment experienced patients (Ickovics \& Meisler, 1997).

The measurement of adherence remains problematic; a standard definition of optimal adherence and completely reliable measures of adherence are lacking. Nevertheless, there has been substantial progress in both of these areas in the past few years. First, it appears that higher levels of adherence are needed for HIV disease than other diseases to achieve the desired therapeutic benefit. Using questionnaires (patient self-reporting and/or face-to-face interview) and electronic compliance monitoring caps (Medication Event Monitoring System [MEMS]), viral suppression is common with at least $54 \%-100 \%$ mean adherence level to antiviral regimens (Bangsberg, 2006). Second, a better appreciation of the value and limitations of different adherence measurements has been addressed (Berg \& Arnsten, 2006; Bova et al., 2005). In AIDS clinical trials, adherence to a medication regimen is currently measured by two major methods: by use of questionnaires and by use of MEMS. The MEMS is considered an objective adherence measure. It consists of a microprocessor in the cap of a medication bottle which records the date and time of bottle opening. The results are downloaded to a computer for analysis. Results demonstrate that medication-taking patterns are highly variable among patients (Kastrissios et al., 1998) and that they often give a more precise measure of adherence than self-report (Arnsten et al., 2001a). However, MEMS data are also subject to error and are not widely available in the clinical setting. Adherence assessment by self-report is usually evaluated by a patient's ability to recall their medication dosing during a specific time interval. Often self-reported measures tend to overestimate HIV medication adherence compared to other methods (Arnsten et al., 2001b, 
Bangsberg et al. 2000; Levine et al., 2006; Liu et al., 2001). Finally, it is important to note that the measurement of viral load levels is of special utility as an indirect measure of adherence in HIV therapeutics. It has been argued that this is not a good adherence measure because other factors may influence viral load (pharmacokinetics, drug resistance etc.). However, there is a tight correlation between viral load and adherence (Haubrich et al., 1999; Paterson et al., 2000), but results vary by adherence method and summary adherence statistic (Vrijens \& Goetghebeur, 1997; Vrijens et al., 2005) . Several recent papers explore the methodological and operational issues when evaluating electronic drug monitoring adherence on viral load (Arnsten et al., 2001b; Fennie et al., 2006; Fletcher et al., 2005; Llabre et al., 2006; Liu et al. , 2006; Liu et al. , 2007; Pearson et al. , 2007; Vrijens et al., 2005). Most importantly, a favorable change in viral load is the desired therapeutic outcome of adherence to HAART.

In this paper, we propose using a viral dynamic model with consideration of long-term medication adherence and drug susceptibility to explore the relationship between adherence to two protease inhibitors, as part of an HAART regimen, and long-term virologic response. In particular, we will use different adherence measures from an AIDS clinical trial study-ACTG398 (Hammer et al., 2002) and compare their performance for predicting virologic response. The dynamic modeling approach (Huang et al., 2006; 2010) allows us to appropriately capture the sophisticated nonlinear relationships and interactions among important factors and virologic response. The complete HIV-1 RNA (viral load) trajectories serve as the virologic response index, which is more informative and sensitive to clinical and drug factors. Thus, this method is more powerful to detect the effect of a clinical or drug factor on the response. Using a Bayesian method (Huang et al., 2006), we fit a long-term viral dynamic model to data from the AIDS clinical trial study to explore the association between adherence and viral load in HIV-infected patients with adjustment of the potential confounding factor, drug susceptibility. In this study, we employed the proposed mechanism-based dynamic model to assess how to efficiently use the adherence data based on questionnaires and the MEMS to predict virologic response. In particular, we intend to address the questions (i) how to summarize the MEMS adherence data for efficient prediction of the virologic response, and (ii) which adherence assessment method, questionnaire or MEMS, is more efficient in predicting the virologic response after accounting for the potential confounding factor, drug resistance. We expect that viral dynamic modeling not only provides a powerful tool to evaluate the effect of adherence on long-term virologic responses, but also can be used to predict antiviral responses for various scenarios that may help with understanding the role of different adherence measure statistics in antiviral activities and assist clinicians in treatment decisions.

\section{Materials}

In this section, we describe the subject population to be studied and observed data to be used in this research. These measurements include RNA viral load, phenotypic drug sensitivity and medication adherence. We also discuss how to evaluate assessment interval lengths and time frames (delay effect of timing) for the MEMS adherence data.

\subsection{Subject population}

The subject sample in our analysis was drawn from the AIDS Clinical Trials Group (ACTG) 398 study (Hammer et al., 2002), a randomized, double-blind, placebo-controlled phase II 
study of amprenavir (APV) as part of several dual protease inhibitor (PI) regimens in subjects with HIV infection in whom initial PI therapy had failed. One of objectives of the ACTG 398 study was to evaluate the genotypic and phenotypic resistance profiles that emerge on treatment and their relationship to the plasma HIV-1 RNA and CD4 cell count responses, and to determine the relationship between drug exposure measured from combined PK and adherence data to the degree and duration of viral response. Subjects in all arms received APV (1200 mg twice a day [q12h]), efavirenz (EFV, $600 \mathrm{mg}$ once a day [qd]), abacavir (300 mg q12h) and adefovir dipivoxil (60 mg qd). A total of 481 subjects were randomized to four treatment arms and received a second PI or placebo: Arm A $(n=116)$ saquinavir (1600 mg q12h); Arm B (n=69) indinavir (1200 mg q12h); Arm C $(n=139)$ nelfinavir (NFV, $1250 \mathrm{mg} \mathrm{q12h);} \mathrm{and} \mathrm{Arm} \mathrm{D}(n=157)$ received a placebo matched for one of these three PIs. Assignment of subjects to treatment arms depended on past PI exposure in the arm. Subjects were scheduled for follow-up visits at study (day 0); at weeks 2, 4, 8, 16 and every 8 weeks thereafter until week 72; and at the time of confirmed virologic failure. More detailed descriptions of this study and study results are given by Hammer et al. (2002) and Pfister et al. (2003). Because phenotype sensitivity testing was performed only on a subset of randomly selected subjects, the number of subjects available for our analysis was greatly reduced. We chose to consider only the subjects within Arm C for our analysis because this arm afforded the greatest number of subjects $(n=31)$ with phenotypic drug susceptibility data on the two PIs (APV and NFV) and had available adherence data, as required for our model. Among these 31 subjects, 13 had phenotypic drug susceptibility data at the time of protocol-defined virologic failure.

\subsection{Observed measurements}

RNA viral load: RNA viral load was measured in copies/mL at study weeks $0,2,4,8$ and every 8 weeks thereafter until week 72 by the ultrasensitive reverse transcriptasepolymerase chain reaction HIV-1 RNA assay. Only measurements taken while on protocoldefined treatment were used in the analysis. All viral load values were log (base 10) transformed. Although, the lower limit of assay quantification was 200 copies/mL, when lower values ( $<200$ copies $/ \mathrm{mL}$ ) were detected, these values were used in the analysis. The exact day of viral load measurement (not predefined study week) was used to compute study day in our analysis.

Medication adherence: Medication adherence was measured by two methods-- by the use of questionnaires and by the use of electronic monitoring caps. Subjects completed an adherence questionnaire (AACTG study 398 questionnaire QL0702) at study weeks 4, 8, 12, 16 , and every 8 weeks thereafter. The questionnaire was completed by the study participant and/or by a face-to-face interview with study personnel. The subject was asked to specify the number of prescribed doses of each drug that he or she had failed to take on each of the preceding 4 days. Questionnaire adherence rates for APV and NFV were determined at each visit as the number of prescribed doses taken divided by the number prescribed doses during the preceding 4 day interval. For electronically monitored adherence, an MEMS cap (Medication Event Monitoring Systems, Aprex Corp., Menlo CA) was used to monitor APV and EFV compliance only. Subjects were asked to bring their medication bottles and caps to the clinic at each study visit (weeks $2,4,8,12,16$, and every 8 weeks thereafter), where cap data were downloaded to computer files and stored for later analysis. Since APV was 
prescribed twice daily, a prescribed AM and PM dosing period was defined for each subject. If a subject opened the bottle at least once during a dosing period, then the subject was recorded as having a positive event $(x=1)$, otherwise $(x=0)$. The MEMS adherence rate for APV was determined as the sum of positive dosing events divided by the sum of prescribed dosing events during the specified time interval. A positive dosing event assumes a presumptive dose. If the MEMS cap was recorded as not in use, the MEMS dosing event was set to missing. In our analysis, we assumed that NFV had the same MEMS adherence rate as APV.

\begin{tabular}{|c|c|c|c|c|}
\hline \multirow[b]{2}{*}{ Case } & \multirow[b]{2}{*}{$\begin{array}{l}\text { MEMS adherence } \\
\text { interval notation }\end{array}$} & \multicolumn{2}{|c|}{ Adherence assessment definition } & \multirow{2}{*}{$\begin{array}{c}\text { Example for day } 56, \\
\text { adherence computed } \\
\text { over }\end{array}$} \\
\hline & & $\begin{array}{l}\text { Interval } \\
\text { length }\end{array}$ & $\begin{array}{l}\text { Time frame length } \\
\text { (weeks to RNA } \\
\text { measurement) }\end{array}$ & \\
\hline 1 & $\mathrm{M}$ & visit time & 0 & Days $28-55$ \\
\hline 2 & M0.1 & 1 week & 0 & Days 49 - 55 \\
\hline 3 & M0.2 & 2 weeks & 0 & Days $42-55$ \\
\hline 4 & M0.3 & 3 weeks & 0 & Days $35-55$ \\
\hline 5 & M1.1 & 1 week & 1 week & Days $43-49$ \\
\hline 6 & M1.2 & 2 weeks & 1 week & Days $36-49$ \\
\hline 7 & M1.3 & 3 weeks & 1 week & Days $29-49$ \\
\hline 8 & M2.1 & 1 week & 2 weeks & Days $36-42$ \\
\hline 9 & M2.2 & 2 weeks & 2 weeks & Days $29-42$ \\
\hline 10 & M2.3 & 3 weeks & 2 weeks & Days $22-42$ \\
\hline 11 & M3.1 & 1 week & 3 weeks & Days 29 - 35 \\
\hline 12 & M3.2 & 2 weeks & 3 weeks & Days $22-35$ \\
\hline 13 & M3.3 & 3 weeks & 3 weeks & Days 15 - 35 \\
\hline
\end{tabular}

Table 1. Summary of the MEMS assessment interval notation and definitions

To determine the best summary metric of the MEMS adherence rate, we evaluated different assessment interval lengths (averaging adherence dosing events over 1, 2, or 3 week intervals) and different assessment time frames (fixing the assessment interval times to end either immediately or 1, 2 or 3 weeks prior to the next measured viral load). Table 1 summarizes the MEMS assessment interval notation and definitions for the 13 models. As an example, M2.2 in Table 1 denotes an MEMS adherence interval length of 2 weeks fixed to end 2 weeks prior to the next viral load measurement; for instance, the MEMS adherence rate for a subject at study week 8 (day 56) was calculated as the number of nominal dosing events divided by the number of prescribed dosing events over study days $29-42$. The case $\mathrm{M}$ serves as a reference and averages all the available MEMS data between viral load measurements.

Phenotypic drug susceptibility: Retrospectively, 200 subjects were randomly selected from the entire ACTG 398 study population for phenotypic sensitivity testing. Of these 139 subjects were tested at baseline based on receiving study treatment for at least 8 weeks and having an available sample. Among these subjects, 59 subjects experienced protocol-defined virologic failure and phenotypic sensitivity testing was performed at the time of failure (Hammer et al., 2002). Phenotypic drug susceptibility was determined by a recombinant virus assay (PhenoSense, ViroLogic, Inc) and values were expressed as the $50 \%$ inhibitory 
concentration $\left(\mathrm{IC}_{50}\right)$ (Molla et al., 1996). All 31 subjects used in our analysis had baseline APV and NFV IC 50 values, of which 13 subjects had follow-up APV and NFV IC 50 values at the time of virologic failure.

\section{Mathematical models and statistical methods}

We fit the dynamic model to the viral load data from 31 subjects with the following considerations. (i) In the model we incorporate the two clinical factors, drug adherence (questionnaire or MEMS) and drug susceptibility (phenotype $\mathrm{IC}_{50}$ values), into a function of treatment efficacy. (ii) We only consider the PI drug effects in the drug efficacy model because the effect of RTI drugs is considered less important compared to the PI drugs and would require a different efficacy model. (iii) We assume that NFV has the same compliance rate as determined for APV by the MEMS method. Details of the mathematical models and statistical methods are described in Huang et al. (2006) and Wu et al. (2005). For completeness, a brief summary of the models and methods is given as follows.

\subsection{Drug resistance model}

As Molla et al. (1996) suggested, the phenotype marker, median inhibitory concentration $\left(\mathrm{IC}_{50}\right)$, can be used to quantify agent-specific drug susceptibility. We use the following model to approximate the within-host changes over time in $\mathrm{IC}_{50}$ (Huang et al., 2003; Huang et al., 2006; Wu et al., 2005).

$$
I C_{50}(t)=\left\{\begin{array}{cc}
I_{0}+\frac{I_{r}-I_{0}}{t_{r}} t & \text { for } 0<t<t_{r}, \\
I_{r} & \text { for } t \geq t_{r},
\end{array}\right.
$$

Where $I_{0}$ and $I_{r}$ are respective values of $I C_{50}(t)$ at baseline and time point $t_{r}$ at which resistant mutations dominate. In our study, $t_{r}$ is the time of virologic failure. For subjects without a failure time $\mathrm{IC}_{50}$, baseline $\mathrm{IC}_{50}$ was held constant over time.

\subsection{Medication adherence model}

Poor adherence to a treatment regimen is one of the major causes of treatment failure. (Ickovics abd Meisler, 1997). The following model is used to represent adherence for a time interval $T_{k}<t \leq T_{k+1}$,

$$
A(t)=\left\{\begin{aligned}
1 & \text { if all doses are taken in }\left(T_{k}, T_{k+1}\right], \\
R_{k} & \text { if } 100 R_{k} \% \text { doses are taken in }\left(T_{k}, T_{k+1}\right],
\end{aligned}\right.
$$

where $0 \leq R_{k}<1$, with $R_{k}$ indicating the adherence rate computed for each assessment interval $\left(T_{k}, T_{k+1}\right]$ based on the questionnaire or MEMS data; $T_{k}$ denotes the adherence assessment time at the $k$ th clinical visit.

\subsection{Drug efficacy model}

In most viral dynamic studies, investigators assumed that either drug efficacy was constant over treatment time (Perelson and Nelson, 1999; Wu and Ding, 1999; Ding and Wu, 2001) or 
antiviral regimens had perfect effect in blocking viral replication (Ho et al., 1995; Perelson et al., 1996, 1997). However, the drug efficacy may change as concentrations of antiretroviral drugs and other factors (e.g. drug resistance) vary during treatment (Dixit et al., 2004). We employ the following modified $E_{\max }$ model (Sheiner, 1985) to represent the time-varying drug efficacy ( see Wu et al. (2005) for more discussion about the drug effect $E_{\max }$ model) for two antiretroviral agents within a class (for example, the two PI drugs APV and NFV),

$$
\gamma(t)=\frac{A_{1}(t) / I C_{50}^{1}(t)+A_{2}(t) / I C_{50}^{2}(t)}{\phi+A_{1}(t) / I C_{50}^{1}(t)+A_{2}(t) / I C_{50}^{2}(t)},
$$

where $I C_{50}^{k}(t) \quad(k=1,2)$ are median inhibitory concentration change over time for the two agents; $A_{k}(t) \quad(k=1,2)$ are adherence profiles of the two drugs measured by questionnaire or the MEMS method. Parameter $\phi$ can be regarded as a conversion factor between in vitro and in vivo $\mathrm{IC}_{50} \mathrm{~s}$ and will be estimated from the data. Note that $\gamma(t)$ ranges from 0 to 1 .

\subsection{Antiviral response model}

We consider a simplified HIV dynamic model with antiviral treatment as follows. (Huang et al., 2006; Wu et al., 2005).

$$
\begin{array}{rlrl}
\frac{d}{d t} T & =\lambda-d_{T} T-[1-\gamma(t)] k T V, \\
\frac{d}{d t} T^{*} & = & {[1-\gamma(t)] k T V-\delta T^{*},} \\
\frac{d}{d t} V & = & N \delta T^{*}-c V,
\end{array}
$$

where the three differential equations represent three compartments: target uninfected cells $(T)$, infected cells $\left(T^{*}\right)$ and free virions $(V)$. Parameter $\lambda$ represents the rate at which new T cells are generated from sources within the body, such as the thymus, $d_{T}$ is the death rate of T cells, $k$ is the infection rate without treatment, $\delta$ is the death rate of infected cells, $N$ is the number of new virions produced from each of infected cell during its life-time, and $c$ is the clearance rate of free virions. The time-varying parameter $\gamma(t)$ is the antiviral drug efficacy at treatment time $t$.

\subsection{Bayesian modeling approach}

Although a number of studies investigated various statistical methods, including Bayesian approaches, of fitting viral dynamic models to predicting virologic responses using shortterm viral load data (Wu and Ding, 1999; Ho et al., 1995; Perelson et al., 1996, 1997; Wu et al., 1999; Notermans et al., 1998; Markowitz et al., 2003; Han et al., 2002), little work has been undertaken to investigate long-term virologic responses. In this paper, we used a hierarchical Bayesian modeling approach (Huang et al., 2006) to estimate the dynamic parameters.

We denote the number of subjects by $n$ and the number of measurements on the $i$ th subject by $m_{i}$. For notational convenience, let $\mu=\left(\ln \phi, \ln c, \ln \delta, \ln \lambda, \ln d_{T}, \ln N, \ln k\right)^{T}$, 
$\Theta=\left\{\theta_{i}, i=1, \cdots, n\right\}, \quad \theta_{i}=\left(\ln \phi_{i}, \ln c_{i}, \ln \delta_{i}, \ln \lambda_{i}, \ln d_{T i}, \ln N_{i}, \ln k_{i}\right)^{T}, \quad \Theta_{\{i\}}=\left\{\theta_{l}, l \neq i\right\} \quad$ and $\mathbf{Y}=\left\{y_{i j}, i=1, \cdots, n ; j=1, \cdots, m_{i}\right\}$. Let $f_{i j}\left(\theta_{i}, t_{j}\right)=\log _{10}\left(V_{i}\left(\theta_{i}, t_{j}\right)\right)$, where $V_{i}\left(\theta_{i}, t_{j}\right)$ denotes the numerical solution of the differential equations (4) for the $i$ th subject at time $t_{j}$. Let $y_{i j}(t)$ and $e_{i}\left(t_{j}\right)$ denote the repeated measurements of common logarithmic viral load and a measurement error with mean zero, respectively. The Bayesian nonlinear mixed-effects model can be written as the following three stages (Davidian and Giltinan, 1995; Huang et al., 2006).

Stage 1. Within-subject variation:

$$
\mathbf{y}_{i}=\mathbf{f}_{i}\left(\theta_{i}\right)+\mathbf{e}_{i}, \quad \mathbf{e}_{i} \mid \sigma^{2}, \theta_{i} \sim N\left(0, \sigma^{2} \mathbf{I}_{m_{i}}\right)
$$

where $\mathbf{y}_{i}=\left(y_{i 1}\left(t_{1}\right), \cdots, y_{i m_{i}}\left(t_{m_{i}}\right)\right)^{T}, \mathbf{f}_{i}\left(\theta_{i}\right)=\left(f_{i 1}\left(\theta_{i}, t_{1}\right), \cdots, f_{i m_{i}}\left(\theta_{i}, t_{m_{i}}\right)\right)^{T}, \mathbf{e}_{i}=\left(e_{i}\left(t_{1}\right), \cdots, e_{i}\left(t_{m_{i}}\right)\right)^{T}$.

Stage 2. Between-subject variation:

$$
\theta_{i}=\mu+\mathbf{b}_{i}, \quad \mathbf{b}_{i} \mid \Sigma \sim N(0, \Sigma)
$$

Stage 3. Hyperprior distributions:

$$
\sigma^{-2} \sim G a(a, b), \quad \mu \sim N(\eta, \Lambda), \quad \Sigma^{-1} \sim W i(\Omega, v)
$$

where the mutually independent Gamma $(G a)$, Normal $(N)$ and Wishart $(W i)$ prior distributions are chosen to facilitate computations (Davidian and Giltinan, 1995). The hyperparameters $a, b, \eta, \Lambda, \Omega$ and $v$ were determined from previous studies and the literature (Perelson and Nelson, 1999; Ho et al., 1995; Perelson et al., 1996, 1997; Nowak and May, 2000). See Huang et al. (2006) for a detailed discussion of the Bayesian modeling approach, including the choice of the hyper-parameters, and the implementation of the Markov chain Monte Carlo (MCMC) procedures (Gamerman, 1997; Wakefield, 1996).

\section{Results}

\subsection{Subject characteristics}

Of the 31 subjects used in our analysis, the mean age was 40 years (SD=7); $94 \%$ were men; and $65 \%$ were white, $23 \%$ black, $10 \%$ Hispanic and 3\% Asian. At baseline, $58 \%$ had prior nonnucleoside reverse transcriptase inhibitor (NNRTI) experience. Median baseline CD4 cell count was 196 cells $/ \mathrm{uL}$ (interquartile range $=120-308$ cells $/ \mathrm{uL}$ ) and median baseline viral load was 38,019 copies $/ \mathrm{mL}$ (interquartile range $=19,498-181,970$ copies $/ \mathrm{mL}$ ). Median time to the last viral load measurement while on protocol-defined treatment was 227 days (interquartile range=168-321 days). Median baseline $\mathrm{IC}_{50}$ values were $21.2 \mathrm{ng} / \mathrm{mL}$ and 38.9 $\mathrm{ng} / \mathrm{mL}$ for $\mathrm{APV}$ and NFV, respectively. Among the 13 subjects with $\mathrm{IC}_{50}$ values at failure time, the median time to virologic failure was 157 days. Overall mean questionnaire adherence rate was 0.95 and 0.96 respectively for APV and NFV and the MEMS adherence rate for APV was 0.80 . Fig. 1 shows the viral load ( $\log _{10}$ transformed) and adherence rates over time based on questionnaire data for APV and NFV drugs and APV MEMS data (13 summary metrics) for one representative subject. 

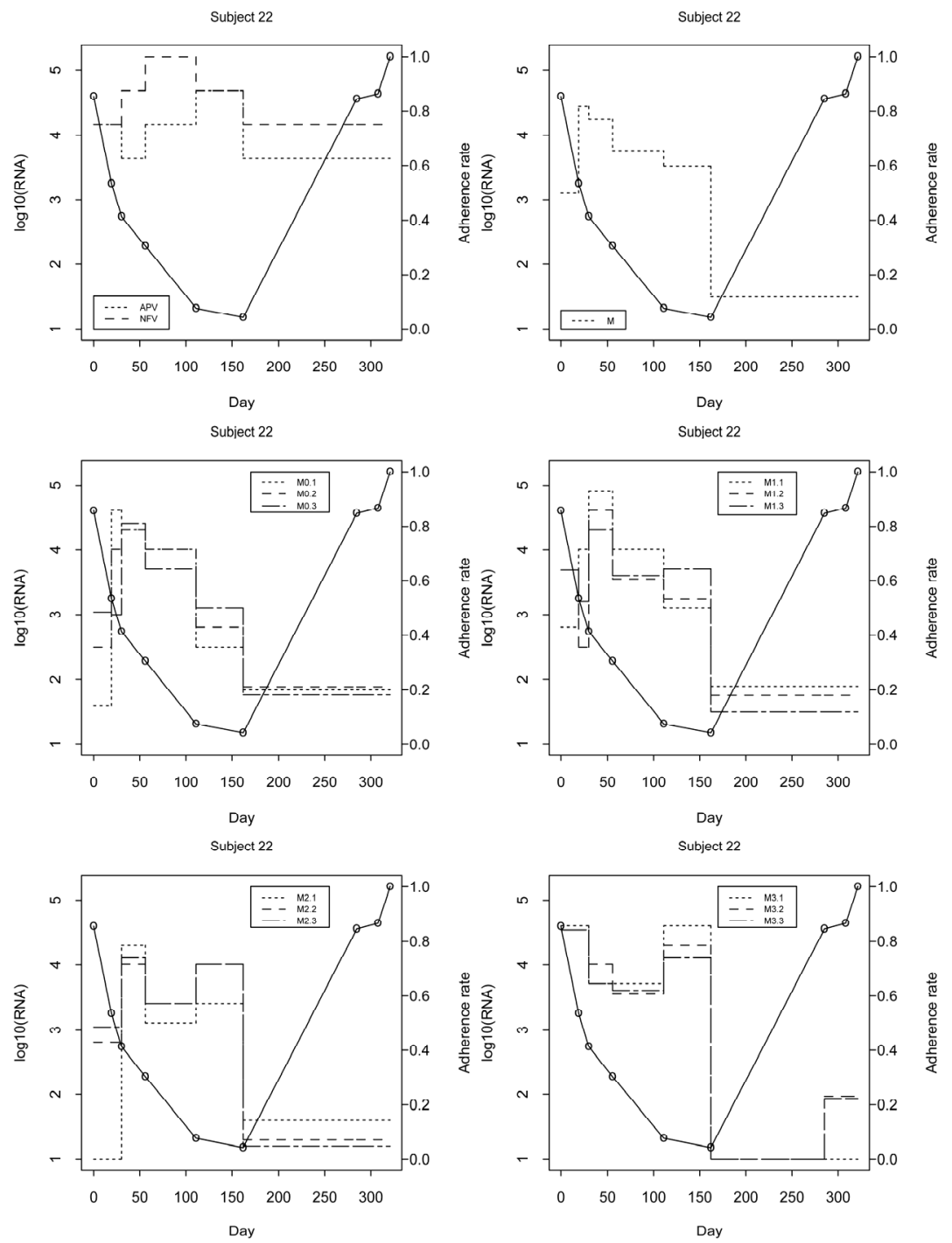

Fig. 1. The trajectories of viral load on $\log _{10}$ scale (solid curves) and adherence rates (stairsteps) over time based on questionnaire data for APV and NFV drugs (upper-left panel) and MEMS data summarized by the 13 models for APV drug (other panels) for one subject

We fit the viral dynamic model to the data from 31 subjects described previously using the proposed Bayesian approach. We incorporated the two clinical factors, drug adherence (questionnaire or MEMS) and drug susceptibility (phenotype $I C_{50}$ values), into a function of treatment efficacy (3). For model fitting and the purpose of comparisons, we set up a control model as the one without using any adherence and drug susceptibility data which 
corresponds to setting $A(t)$ and $I C_{50}(t)$ to be 1 in Eq. (3), i.e., $\gamma(t)=2 /(\phi+2)$. Other 14 models are specified based on the combination of drug susceptibility $\left(I C_{50}\right)$ data and 14 different adherence summary metrics (1 questionnaire and 13 MEMS summary metrics listed in Table 1). Note that the abbreviation IM2.2, for example, denotes the model incorporating the data of drug resistance (I) and MEMS adherence rate (M2.2) summarized as an interval length of 2 weeks fixed to end 2 weeks prior to the next viral load measurement. For example, the MEMS adherence rate for a subject at study week 8 (day 56) was calculated over a 14 day interval from study days 29-42 and this value was used to represent adherence from the previous study visit to the study visit at day 56 for modeling fitting.

\subsection{Model fitting}

In order to assess how adherence rates, determined from 14 different scenarios, interact with drug susceptibility to contribute to virologic response, we fitted the models to all 14 scenarios as well as the control model and compared the fitting results. We found that, overall, the model with adherence rate determined from MEMS dosing events averaged over a 2 week assessment interval either 1 week prior to a viral load measurement (IM1.2) or 2 weeks prior to a viral load measurement (IM2.2) provided the best fits to the observed data, compared to the other 13 models for most subjects; the control model, lacking factors for subject-specific drug adherence and susceptibility, failed to fit viral load rebounds and
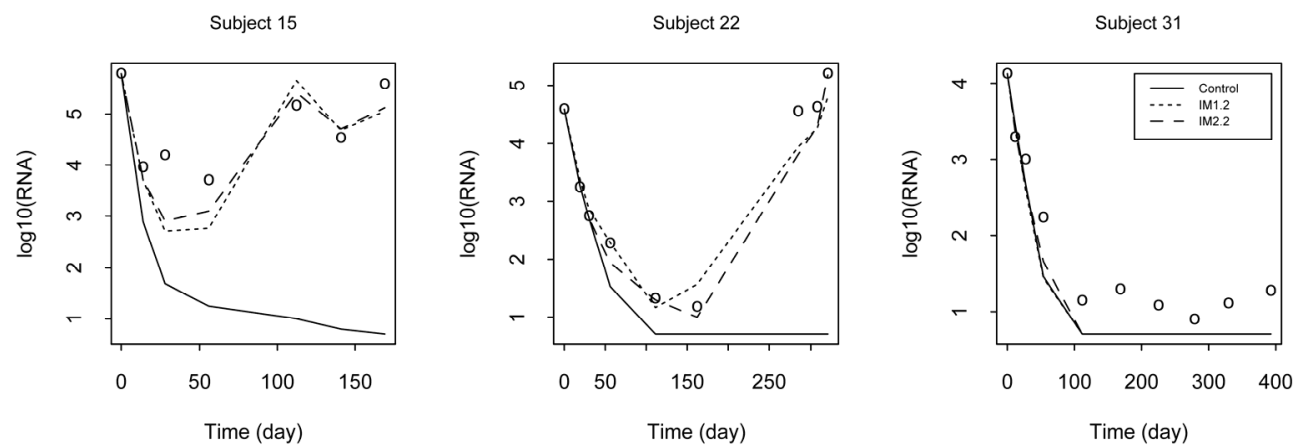

Fig. 2. The estimated viral trajectory for three representative subjects from the model fitting: (i) Control model (solid curves), (ii) IM1.2 (dotted curves) and (iii) IM2.2 (dashed curves). The observed values are indicated by circles.

fluctuations and provided the worst fitting results for the majority of subjects. For the purpose of illustration, the model fitting curves for three representative subjects from the control model (solid curves), the IM1.2 model (dotted curves), and the IM2.2 model (dashed curves) are displayed in Fig. 2.

\subsection{Individual dynamics parameter estimates}

Table 2 presents the results of estimated dynamic parameters for individual subjects and the sample summary statistics (minimum, median, mean, maximum, standard deviation (SD) and coefficient of variation (CV) for the model IM2.2 that provided the best fit to the observed data. We can see from Table 2 a relatively large between-subject variation in the 
Antiviral Drugs - Aspects of Clinical Use and Recent Advances

\begin{tabular}{|c|c|c|c|c|c|c|c|}
\hline Subject & $\phi_{i}$ & $c_{i}$ & $\delta_{i}$ & $\lambda_{i}$ & $d_{T i}$ & $N_{i}$ & $k_{i} \times 10^{4}$ \\
\hline 1 & 0.002 & 3.144 & 0.105 & 68.830 & 0.041 & 126.353 & 2.083 \\
\hline 2 & 0.002 & 3.366 & 0.203 & 193.493 & 0.021 & 763.481 & 0.800 \\
\hline 3 & 0.002 & 3.065 & 0.228 & 103.261 & 0.051 & 516.932 & 1.363 \\
\hline 4 & 0.001 & 3.963 & 0.294 & 67.549 & 0.110 & 709.988 & 0.738 \\
\hline 5 & 0.002 & 2.499 & 1.238 & 171.962 & 0.142 & 9384.339 & 0.868 \\
\hline 6 & 0.002 & 3.219 & 0.224 & 108.737 & 0.056 & 495.182 & 1.526 \\
\hline 7 & 0.002 & 3.631 & 0.078 & 111.508 & 0.017 & 109.594 & 1.549 \\
\hline 8 & 0.001 & 4.103 & 0.157 & 73.195 & 0.050 & 354.894 & 1.124 \\
\hline 9 & 0.002 & 2.286 & 0.809 & 177.204 & 0.097 & 4070.423 & 1.112 \\
\hline 10 & 0.002 & 2.620 & 1.058 & 208.394 & 0.091 & 6031.472 & 1.033 \\
\hline 11 & 0.002 & 3.213 & 0.219 & 83.127 & 0.058 & 479.131 & 1.316 \\
\hline 12 & 0.002 & 2.804 & 0.315 & 96.976 & 0.071 & 806.579 & 1.622 \\
\hline 13 & 0.001 & 3.705 & 0.164 & 88.307 & 0.056 & 581.903 & 0.727 \\
\hline 14 & 0.002 & 2.877 & 0.132 & 130.303 & 0.026 & 341.015 & 1.347 \\
\hline 15 & 0.003 & 2.316 & 0.434 & 207.091 & 0.043 & 2197.731 & 1.064 \\
\hline 16 & 0.003 & 1.753 & 0.924 & 160.891 & 0.120 & 4224.349 & 1.867 \\
\hline 17 & 0.001 & 4.041 & 0.211 & 127.465 & 0.023 & 765.045 & 0.692 \\
\hline 18 & 0.002 & 3.367 & 0.216 & 85.716 & 0.051 & 508.451 & 1.148 \\
\hline 19 & 0.002 & 3.955 & 0.114 & 74.292 & 0.033 & 164.046 & 1.498 \\
\hline 20 & 0.001 & 3.938 & 0.116 & 117.911 & 0.023 & 356.109 & 0.801 \\
\hline 21 & 0.001 & 2.887 & 0.314 & 306.351 & 0.019 & 1636.315 & 0.486 \\
\hline 22 & 0.003 & 2.003 & 0.569 & 100.966 & 0.067 & 1186.222 & 0.654 \\
\hline 23 & 0.001 & 4.273 & 0.260 & 43.015 & 0.136 & 474.269 & 1.258 \\
\hline 24 & 0.001 & 3.506 & 0.131 & 157.873 & 0.028 & 643.514 & 0.847 \\
\hline 25 & 0.002 & 2.277 & 0.839 & 174.871 & 0.105 & 4912.339 & 1.189 \\
\hline 26 & 0.001 & 3.847 & 0.340 & 54.983 & 0.135 & 714.569 & 1.116 \\
\hline 27 & 0.003 & 2.730 & 0.218 & 103.326 & 0.042 & 411.427 & 1.690 \\
\hline 28 & 0.002 & 3.510 & 0.073 & 133.204 & 0.015 & 106.627 & 1.366 \\
\hline 29 & 0.002 & 3.751 & 0.186 & 108.477 & 0.026 & 435.276 & 1.111 \\
\hline 30 & 0.002 & 3.760 & 0.162 & 96.575 & 0.031 & 354.927 & 1.192 \\
\hline 31 & 0.002 & 3.415 & 0.144 & 112.288 & 0.034 & 392.298 & 1.140 \\
\hline Min & 0.001 & 1.753 & 0.073 & 43.015 & 0.015 & 106.627 & 0.486 \\
\hline Med & 0.002 & 3.367 & 0.218 & 108.737 & 0.050 & 516.932 & 1.140 \\
\hline Max & 0.003 & 4.273 & 1.238 & 306.351 & 0.142 & 9384.339 & 2.083 \\
\hline Mean & 0.0017 & 3.285 & 0.328 & 124.134 & 0.059 & 1427.574 & 1.172 \\
\hline SD & 0.0006 & 0.647 & 0.305 & 55.726 & 0.039 & 2118.271 & 0.373 \\
\hline CV (\%) & 33.037 & 19.686 & 93.066 & 44.892 & 66.245 & 148.383 & 31.801 \\
\hline
\end{tabular}

Table 2. The estimated dynamic parameters from the IM2.2 model for individual subjects, where Min, Med, Max, SD and CV=SD/Mean denote the minimum, median, maximum, standard deviation and coefficient of variation, respectively. 
seven viral dynamic parameters was observed (CV ranges from $20 \%$ to $148 \%$ ) among the 31 subjects. Generally speaking, the virologically successful subjects (maintaining plasma HIV1 RNA levels of less than 200 copies/mL) have higher clearance rates of free virions $(c)$, but smaller efficacy parameter estimates $(\phi)$, and lower death rates of infected cells $(\delta)$; these results show the similar patterns to those displayed in Figure 4 studied by Wu et al. (2005) The individual parameter estimates from both the IM2.2 and IM1.2 models are significantly correlated for all seven parameters, while the individual parameter estimates for the control model appear significantly different from those for the model IM2.2 for most of the seven parameters (data not shown here).

\subsection{Effects of adherence rate determined by questionnaire vs. MEMS data}

In order to assess how different adherence rates measured by questionnaires and MEMS contribute to the virologic response, we compared the fitting results of models with all 14 adherence scenarios and the control model. The mean of the sum of the squared deviations (SSD) was used to assess model fit and the SSD was calculated by $\sum_{j=1}^{m_{i}}\left(y_{i j}-\hat{y}_{i j}\right)^{2}$ for each subject, where $y_{i j}$ and $\hat{y}_{i j}$ are the observed and predicted values, respectively. The mean SSDs are plotted for all the models in Fig. 3, with the best fitting models having a smaller mean SSD, and sign test $p$-values from pairwise comparisons are reported in Table 3.

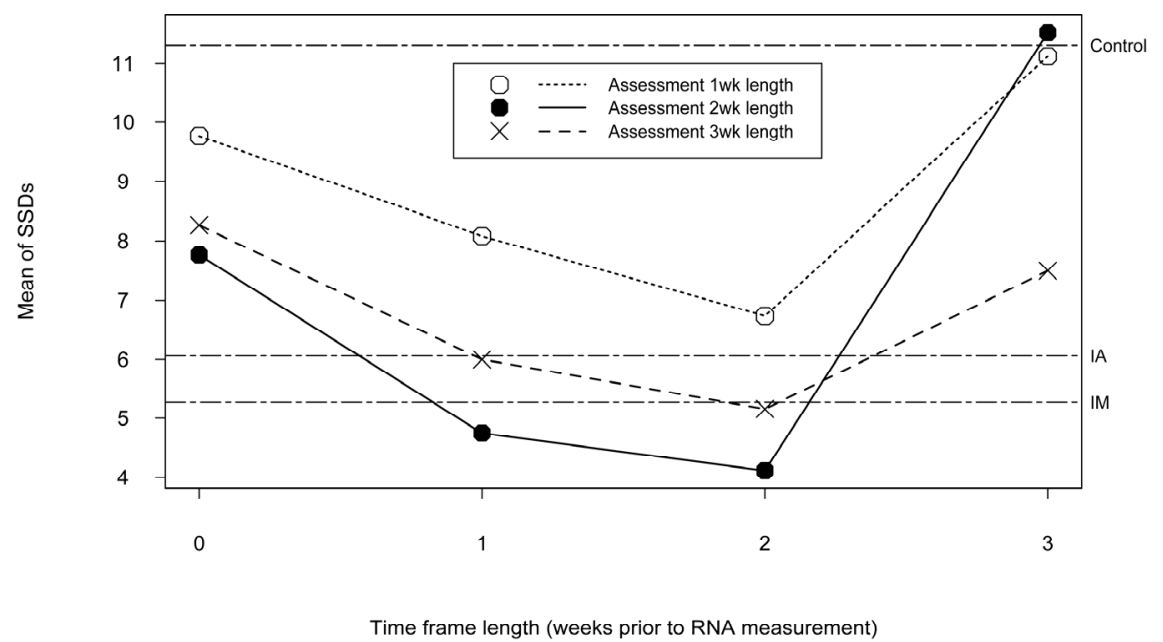

Fig. 3. Comparison of mean of SSDs for models from the 14 different determinants of adherence with drug resistance and the control model. The three horizontal lines represent mean of the SSDs for control, IA and IM models, respectively.

\subsection{What MEMS assessment interval length is best?}

The pattern in Fig. 3 shows that when the time frame for MEMS assessment is fixed, models with a 2 week MEMS assessment interval length generally outperform models with an assessment interval length of 1 or 3 weeks. 


\begin{tabular}{|c|c|c|c|c|c|c|c|c|c|c|c|c|c|c|}
\hline Model & Control & IA & IM & IM0.1 & IM0.2 & IM0.3 & IM1.1 & IM1.2 & IM1.3 & IM2.1 & IM2.2 & IM2.3 & IM3.1 & IM3.2 \\
\hline IA & $<0.001$ & & & & & & & & & & & & & \\
\hline IM & $<0.001$ & 0.106 & & & & & & & & & & & & \\
\hline IM0.1 & 0.106 & 0.007 & 0.002 & & & & & & & & & & & \\
\hline IM0.2 & 0.019 & 0.209 & 0.007 & 0.020 & & & & & & & & & & \\
\hline IM0.3 & 0.048 & 0.048 & 0.020 & 0.106 & 0.369 & & & & & & & & & \\
\hline IM1.1 & 0.001 & 0.590 & 0.020 & 0.106 & 0.209 & 0.858 & & & & & & & & \\
\hline IM1.2 & $<0.001$ & 0.048 & 0.029 & 0.002 & 0.007 & 0.007 & 0.007 & & & & & & & \\
\hline IM1.3 & 0.001 & 0.858 & 0.858 & 0.048 & 0.048 & 0.048 & 0.209 & 0.048 & & & & & & \\
\hline IM2.1 & $<0.001$ & 0.106 & 0.209 & 0.020 & 0.007 & 0.048 & 0.048 & 0.048 & 0.209 & & & & & \\
\hline IM2.2 & $<0.001$ & 0.007 & 0.020 & $<0.001$ & 0.001 & $<0.001$ & 0.048 & 0.590 & 0.048 & 0.020 & & & & \\
\hline IM2.3 & $<0.001$ & 0.369 & 0.209 & 0.011 & 0.002 & 0.002 & 0.209 & 0.106 & 0.858 & 0.590 & 0.048 & & & \\
\hline IM3.1 & 0.106 & 0.007 & $<0.001$ & 0.369 & 0.590 & 0.048 & 0.007 & $<0.001$ & 0.007 & 0.001 & 0.002 & 0.002 & & \\
\hline IM3.2 & 0.106 & 0.209 & 0.002 & 0.858 & 0.858 & 0.007 & 0.007 & 0.001 & 0.002 & 0.007 & 0.007 & 0.002 & 0.048 & \\
\hline IM3.3 & 0.001 & 0.858 & 0.020 & 0.048 & 0.020 & 0.369 & 0.209 & 0.007 & 0.369 & 0.369 & 0.001 & 0.020 & 0.002 & 0.020 \\
\hline MSSD & 11.30 & 6.06 & 5.27 & 9.77 & 7.77 & 8.27 & 8.09 & 4.75 & 6.00 & 6.73 & 4.11 & 5.16 & 11.12 & 11.56 \\
\hline
\end{tabular}

Table 3. Pairwise comparisons of sum of squared deviations (SSD) from individual subjects for 15 models. The $p$-values were obtained using the sign test and MSSD is the mean of SSD

\subsection{What MEMS assessment time frame (delay effect of timing) is best?}

As seen in Fig. 3, regardless of the assessment interval length, models which assess compliance 2 weeks prior to viral load generally outperform models which assess compliance immediately before viral load, 1 week before or 3 weeks before viral load measurement. Overall, the model with a MEMS assessment interval length of 2 weeks measured from 4 to 2 weeks prior to viral load measurement (IM2.2) was significantly a better predicator of viral load over time than any other models, with the exception of the IM1.2 model.

From Table 3, the means and standard deviations of the SSDs for the models based on IM1.2 $(4.75 \pm 5.38)$ and IM2.2 $(4.11 \pm 4.18)$ were significantly less than those of the other 13 models. We can see that that the IM1.2 and IM2.2 models were significantly better than the models based on the other 13 models $(p \leq 0.001 \sim 0.048)$, but they were not significantly different each other $(p=0.590)$. The control model was significantly worse than those based on all other models ( $p \leq 0.001 \sim 0.020)$ except for the 2 models (IM3.1 and IM3.2: $p=0.106$ ).

\subsection{What adherence assessment method (questionnaire vs MEMS) is best?}

Further, we compared the model fittings with all possible combinations of $\mathrm{IC}_{50}$ and the four determinants of adherence (A, M, M1.2 and M2.2). The mean of SSD for all the 10 models is plotted in Figure 4, and sign test $p$-values from pairwise comparisons are reported in Table 4 . The results indicate that (i) the control model was significantly worse than those based on all other 9 models $(p \leq 0.001 \sim 0.007)$; (ii) the models IM1.2 and IM2.2 were significantly better than the other eight models ( $p \leq 0.001 \sim 0.048$ ); (iii) the models I, A, M, M1.2, M2.2, IA and IM do not provide significantly different results $(p=0.048 \sim 0.858)$ except for two marginally significant results. In particular, the models IA and IM are not better than the model I $(p=0.209,0.590)$, and the models IM1.2 and IM2.2 are significantly better than the models I, M1.2 and M2.2 ( $p=0.007 \sim 0.048)$. Overall, adherence assessed by an optimal summary MEMS metric with the confounding resistance factor combinations (IM1.2 and IM2.2) was a 
better predictor of virologic response than adherence assessed by questionnaires, MEMS alone or two-factor combinations.

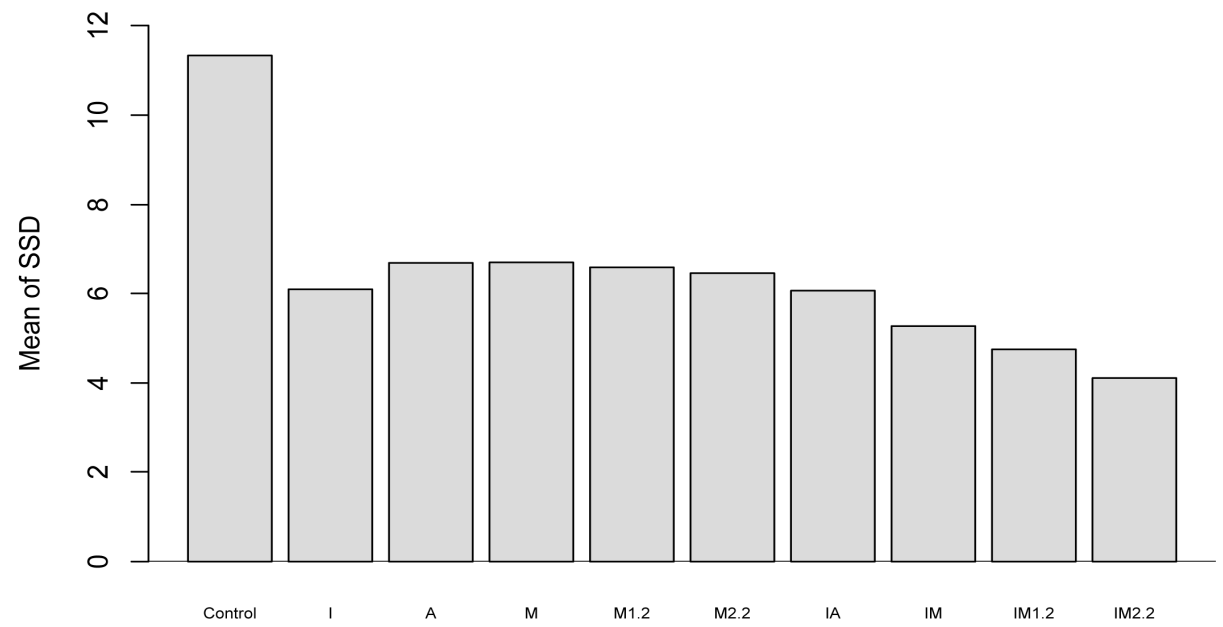

Fig. 4. Comparison of SSDs for the models from 9 different determinants of adherence and/or drug resistance as well as the control model

\begin{tabular}{llllllllllll}
\hline \hline & Models & Control & I & A & M & M1.2 & M2.2 & IA & IM & IM1.2 & IM2.2 \\
\hline \multirow{6}{*}{$p$} & I & $<0.001$ & & & & & & & & & \\
& A & 0.007 & 0.106 & & & & & & & & \\
M & 0.007 & 0.106 & 0.858 & & & & & & & \\
& M1.2 & $<0.001$ & 0.106 & 0.590 & 0.858 & & & & & & \\
& M2.2 & $<0.001$ & 0.209 & 0.858 & 0.858 & 0.369 & & & & & \\
& IA & $<0.001$ & 0.209 & 0.209 & 0.590 & 0.209 & 0.590 & & & & \\
& IM & $<0.001$ & 0.590 & 0.209 & 0.048 & 0.048 & 0.209 & 0.106 & & & \\
& IM1.2 & $<0.001$ & 0.048 & 0.002 & 0.002 & 0.020 & 0.048 & 0.048 & 0.026 & & \\
& IM2.2 & $<0.001$ & 0.048 & 0.002 & 0.002 & 0.007 & 0.048 & 0.007 & 0.020 & 0.590 & \\
\hline SSD & Mean & 11.30 & 6.09 & 6.68 & 6.69 & 6.68 & 6.45 & 6.06 & 5.27 & 4.75 & 4.11 \\
& \pm SD & 9.28 & 6.84 & 6.02 & 6.87 & 6.79 & 6.33 & 5.66 & 5.52 & 5.38 & 4.18 \\
\hline
\end{tabular}

Table 4. Pairwise comparisons of sum of squared deviations (SSD) from individual subjects for 10 models. The $p$-values were obtained using the sign test.

\section{Conclusion and discussion}

Several studies investigated the association between virologic responses and adherence assessed by MEMS data only without considering other confounding factors such as drug resistance using standard modeling methods including Poisson regression (Knafl et al., 2004), logistic regression (Vrijens et al., 2005) and linear mixed-effects model (Liu et al., 2007). In this article, we developed a mechanism-based nonlinear time-varying differential equation model for long-term dynamics to (i) establish the relationship of virologic response (viral load trajectory) with drug adherence and drug resistance, (ii) to describe both suppression and resurgence of virus, (iii) to directly incorporate observed drug adherence 
and susceptibility into a function of treatment efficacy and (iv) to use a hierarchical Bayesian mixed-effects modeling approach that can not only combine prior information with current clinical data for estimating dynamic parameters, but also characterize inter-subject variability. Our modeling approach allows us to estimate time-varying antiretroviral efficacy during the entire course of a treatment regimen by incorporating the information of drug exposure and drug susceptibility. Thus, the results of estimated dynamic parameters based on this model should be more reliable and reasonable to interpret long-term HIV dynamics. Our models are simplified with the main goals of retaining crucial features of HIV dynamics and, at the same time, guaranteeing their applicability to typical clinical data, in particular, long-term viral load measurements.

We employed the proposed mechanism-based dynamic model to assess how to efficiently use adherence rates based on questionnaires and MEMS dosing events to predict virologic response. In particular, we intended to address the questions (i) how to summarize the MEMS adherence data for efficient prediction of virologic response, and (ii) which adherence assessment method, questionnaire or MEMS, is a more efficient predictor of virologic response after accounting for potential confounding factors such as drug resistance between subjects.

For the MEMS data, we found that the best summary metric for prediction of virologic response in terms of model fitting residuals (prediction error) is the adherence rate determined from MEMS dosing events averaged over a 2 week assessment interval, 1 week or 2 weeks prior to the next measured RNA observation (denoted by IM1.2 or IM2.2). The model fitting residuals from both models (IM2.2 and IM1.2) are significantly smaller than any other 13 models $(p \leq 0.001 \sim 0.048)$, but they were not significantly different each other $(p=0.590)$.

The model which used all available MEMS data between study visits to determine the adherence rate (the standard analysis) did not perform significantly better in terms of prediction of virologic response compared to the model with questionnaire adherence data $(p=0.106)$.

We also compared the model fittings with all possible combinations of $\mathrm{IC}_{50}$ and the four determinants of adherence data (see Fig. 4). The results indicate that (i) the control model was significantly worse than those based on all other 9 models $(p \leq 0.001 \sim 0.007)$; (ii) the models IM1.2 and IM2.2 were significantly better than the eight other models ( $p \leq 0.001 \sim 0.048$ ); (iii) the models I, A, M, M1.2, M2.2, IA and IM do not provide significantly different results $(p=0.048 \sim 0.858)$ except for two marginally significant results. In particular, the models IA and IM did not improve upon the model I, which indicates that adherence measured by questionnaire and MEMS dosing events averaged over study visit interval did not provide any additional information to drug susceptibility in predicting virologic response. However, the models IM1.2 and IM2.2 did outperform the models I, M1.2 and M2.2, which indicates that the combination of drug susceptibility and adherence assessed over 2 week interval measured from 1 or 2 weeks prior to a RNA measurement provided significant additional information compared to either drug susceptibility or adherence alone in predicting virologic response.

Although the analysis presented here used a simplified model, which appeared to perform well in capturing and explaining the observed patterns, and characterizing the biological mechanisms of HIV infection under relatively complex clinical situations, some limitations 
exist for the proposed modeling method. Firstly, our model is a simplified model and there are many possible variations (Perelson and Nelson, 1999; Nowak and May, 2000; Callaway and Perelson, 2002). We did not separately consider the compartments of short-lived productively infected cells, long-lived and latently infected cells.(Perelson et al., 1997). Instead we examined a pooled productively infected cell population. The virus compartment was not further decomposed into infectious virions and non-infectious virions as in the paper by Perelson et al. (1996). Thus, different mechanisms of RTI and PI drug effects were not modeled. In fact, we only considered PI drug effects in the drug efficacy model (3) since the RTI drugs have a different adherence-resistance relationship. Further studies will be conducted in considering both PI and RTI drug effects in the models. Secondly, the availability of $\mathrm{IC}_{50}$ data was limited to baseline and failure time, as is typical in clinical trials. Thus, we extrapolated the $\mathrm{IC}_{50}$ data linearly to the whole treatment period in our modeling. The linear extrapolation is the best approximation that we can get from the sparse $I C_{50}$ data (Wu et al., 2005). The linear assumption might have some influence on the estimation results since the $I C_{50}$ might have jumped to a higher level earlier before the failure time when we obtained the sample for drug resistance test. However, we expect that this assumption had little effect on the prediction of virologic response since we had relatively frequent monitoring (monthly in the later stage) of virologic failure in this study. Thirdly, a more complete model of antiretroviral treatment efficacy would ideally also consider the time-varying function of concentrations of drug in plasma (Huang et al., 2003). Unfortunately, the limited availability of drug concentration data prohibited our inclusion of PK parameters in our model. Lastly, as measurements of adherence may not reflect actual adherence profiles for individual patients, the data quality would affect our estimation results for viral dynamic parameters. For example, adherence data measured by questionnaires may not be accurate. More accurate measurements of the MEMS adherence data were used in this paper and it was found that the MEMS adherence data can provide a better prediction of virologic response compared with the questionnaire adherence data, when the MEMS data are summarized in an appropriate way. Further studies on these issues are definitely needed. Nevertheless, these limitations would not offset the major findings from our modeling approach, although further improvement may be warranted.

In summary, MEMS adherence data may not be correlated better to virologic response compared to questionnaire adherence data unless the MEMS cap data are summarized in an appropriate way where adherence was assessed over 2 week interval measured from 1 or 2 weeks prior to a RNA measurement in our case. Our study also shows that the mechanismbased dynamic model is powerful and effective to establish a relationship of antiviral response with drug exposure and drug susceptibility.

\section{Acknowledgment}

The author would like to thank the ACTG398 study team for allowing me to use the clinical data from their study. This research was partially supported by NIAID/NIH grant AI080338 and MSP/NSA grant H98230-09-1-0053.

\section{References}

Arnsten, J.; Demas, P. \& Gourvetch, M.; et al. (February, 2001a). Adherence and viral load in HIV infected drug users: Comparison of self-report and medication event monitors (MEMS). 8th CROI. Chicago, USA 
Arnsten, J. H.; Demas, P. A.; Farzadegan, H.; et al. (2001b). Antiretroviral therapy adherence and viral suppression in HIV-infected drug users: comparison of self-report and electronic monitoring. Clinical Infectious Diseases, Vol.33, pp.1417-1423

Bangsberg, D. R.; Hecht, F. M.; Charlebois, E. D.; et al. (March, 2000). Adherence to protease inhibitors, HIV-1 viral load, and development of drug resistance in an indigent population. AIDS, Vol.14, No.4, pp.357-366

Bangsberg, D. R. (2006). Less than 95\% adherence to nonnucleoside reverse-transcriptase inhibitor therapy can lead to viral suppression. Clinical Infectious Diseases, Vol.43, pp.939-941

Berg, K. M. \& Arnsten, J. H. (2006). Practical and conceptual challenges in measuring antiretroviral adherence. J Acquir Immune Defic Syndr., Vol.43, pp.S79-S87

Bova, C. A.; Fennie, K. P.; Knafl, G. J.; et al. (2005). Use of electronic monitoring devices to measure antiretroviral adherence: practical considerations. AIDS and Behavior, Vol.9, pp.103-110

Callaway, D. S. \& Perelson, A. S. (2002). HIV-1 infection and low steady state viral loads. Bull. Math. Biol., Vol.64, pp.29-64

Centers for Disease Control and Prevention (CDC). (2009). HIV/AIDS Surveillance Report, pp.1-44, Atlanta, GA, USA

Davidian, M. \& Giltinan, D. M. (Eds). (1005). Nonlinear Models for Repeated Measurement Data. Chapman $\mathcal{E}$ Hall, London

Ding, A. A. \& Wu, H. (2001). Assessing antiviral potency of anti-HIV therapies in vivo by comparing viral decay rates in viral dynamic models. Biostatistics. Vol.2, pp.13-29

Dixit, N. M.; Markowitz, M.; Ho DD; et al. (2004). Estimates of intracellular delay and average drug efficacy from viral load data of HIV-infected individuals under antiretroviral therapy. Antiviral Therapy. Vol.9, pp.237-246

Fennie, K. P.; Bova, C. A. \& Williams, A. B. (2006). Adjusting and censoring electronic monitoring device data. Implications for study outcomes. J Acquir Immune Defic Syndr., Vol.43, pp.S88-S95

Fletcher, C. V.; Testa, M. A.; Brundage, R. C.; et al. (2005). Four measures of antiretroviral medication adherence and virologic response in AIDS clinical trials group study 359. J Acquir Immune Defic Syndr. , Vol.40, pp.301-306

Gamerman, D. (1997). Markov Chain Monte Carlo: Stochastic Simulation for Bayesian Inference. Chapman $\mathcal{E}$ Hall, London

Hammer, S. M.; Vaida, F.; Bennett, K. K.; et al. (2002). Dual vs single protease inhibitor therapy following antiretroviral treatment failure: a randomized trial. JAMA, Vol.288, pp.169-180

Han, C.; Chaloner, K. \& Perelson, A. S. (2002). Bayesian analysis of a population HIV dynamic model. Case Studies in Bayesian Statistics, Vol. 6. Springer-Verlag, New York

Haubrich, R. H.; Little, S. J.; Currier, J. S.; et al. (1999). The value of patient-reported adherence to antiretroviral therapy in predicting virologic and immunologic response. AIDS, Vol.13, pp.1099-1107

Ho, D. D.; Neumann, A. U.; Perelson, A. S.; et al. (1995). Rapid turnover of plasma virions and CD4 lymphocytes in HIV-1 infection. Nature, Vol.373, pp.123-126 
Huang, Y.; Rosenkranz, S. L. \& Wu, H. (2003). Modeling HIV dynamics and antiviral responses with consideration of time-varying drug exposures, sensitivities and adherence. Math. Biosci., Vol.184, pp.165-86

Huang, Y.; Liu, D. \& Wu, H. (2006). Hierarchical Bayesian methods for estimation of parameters in a longitudinal HIV dynamic system. Biometrics, Vol.62, pp.413-423

Huang, Y., Wu, H. \& Acosta, P. E. (2010). Hierarchical Bayesian inference for HIV dynamic differential equation models incorporating multiple treatment factors. Biometrical Journal, Vol.52, No.4, pp.470-486

Ickovics, J. R. \& Meisler, A. W. (1997). Adherence in AIDS clinical trial: a framework for clinical research and clinical care. J Clin Epidemiol., Vol., pp.385-391

Kastrissios, H.; Suarez, J. R.; Katzenstein, D.; et al. (1998). Characterizing patterns of drugtaking behavior with a multiple drug regimen in an AIDS clinical trial. AIDS, Vol.12, pp.2295-2303

Knafl, G. J.; Fennie, K. P.; Bova, C.; et al. (2004). Electronic monitoring device event modeling on an individual-subject basis using adaptive Poisson regression. Statistics in Medicine, Vol.23, pp.783-801

Levine, A. J.; Hinkin, C. H.; Marion, S.; et al. (2006). Adherence to antiretroviral medications in HIV: differences in data collected via self-report and electronic monitoring. Health Psychol. , Vol.25, pp.329-35

Llabre, M. M.; Weaver, K. E.; Duran, R. E.; et al.(2006). A measurement model of medication adherence to highly active antiretroviral therapy and its relation to viral load in HIV-positive adults. AIDS Patient Care STDS., Vol.20, pp.701-711

Liu, H.; Golin, C. E.; Miller, L. G.; et al. (2001). A comparison study of multiple measures of adherence to HIV protease inhibitors. Ann Intern Med. , Vol.134, pp.968-77

Liu, H.; Miller, L. G.; Hays, R. D.; et al. (2006). Repeated measures longitudinal analyses of HIV virologic response as a function of percent adherence, dose timing, genotypic sensitivity, and other factors. J Acquir Immune Defic Syndr. , Vol.41, pp.315-322

Liu, H.; Miller, L. G.; Golin, C.E.; et al. (2007). Repeated measures analyses of dose timing of antiretroviral medication and its relationship to HIV virologic outcomes. Statistics in Medicine, Vol.26, pp.991-1007

Markowitz, M.; Louie, M.; Hurley, A.; et al. (2003). A novel antiviral intervention results in more accurate assessment of human immunodeficiency virus type 1 replication dynamics and T-cell decay in vivo. Journal of Virology, Vol.77, pp.5037-5038

Molla, A.; Korneyeva, M.; Gao, Q.; et al. (1996). Ordered accumulation of mutations in HIV protease confers resistance to ritonavir. Nat Med., Vol.2, pp.760-766

Nowak, M. A. \& May, R.M. (Eds). (2000). Virus Dynamics: Mathematical Principles of Immunology and Virology. Oxford University Press, Oxford

Notermans, D. W.; Goudsmit, J.; Danner, S.A.; et al. (1998). Rate of HIV-1 decline following antiretroviral therapy is related to viral load at baseline and drug regimen. J Acquir Immune Defic Syndr., Vol.12, pp.1483-1490

Palella, F. J.; Delaney, K. M; Moorman, A. C.; et al. (1998). Declining morbidity and mortality among patients with advanced human immunodeficiency virus infection. The New England Journal of Medicine, Vol.338, No.13, pp. 853-860, ISSN 0028-4793

Paterson, D. L.; Swindells, S.; Mohr, J.; et al. (2000). Adherence to protease inhibitor therapy and outcomes in patients with HIV infection. J Intern Med., Vol.133, pp.21-30 
Pearson, C. R.; Simoni, J. M.; Hoff, P.; et al. (2007). Assessing antiretroviral adherence via electronic drug monitoring and self-report: an examination of key methodological issues. AIDS and Behavior, Vol.11, pp.161-173

Perelson, A. S.; Neumann, A. U.; Markowitz, M.; et al. (1996). HIV-1 dynamics in vivo: virion clearance rate, infected cell life-span, and viral generation time. Science, Vol.271, pp.1582-1586

Perelson, A. S.; Essunger, P.; Cao, Y.; et al. (1997). Decay characteristics of HIV-1-infected compartments during combination therapy. Nature, Vol.387, pp.188-191

Perelson, A. S. \& Nelson, P. W. (1999). Mathematical analysis of HIV-1 dynamics in vivo. SIAM Review, Vol.41, pp.3-44

Pfister, M.; Labbé, L.; Hammer, S. M.; et al. (2003). Population Pharmacokinetics and Pharmacodynamics of Efavirenz, Nelfinavir, and Indinavir: Adult AIDS Clinical Trial Group Study 398. Antimicrob Agents Chemoter., Vol.47, pp130-137

Sheiner, L. B. (1985). Modeling pharmacodynamics: parametric and nonparametric approaches. In: Rowland $\mathrm{M}$, et al, eds. Variability in Drug Therapy: Description, Estimation, and Control. pp.139-152, Raven Press, New York.

Vrijens, B. \& Goetghebeur, E. (1997). Comparing compliance patterns between randomized treatments. Control Clin Trials., Vol.18, pp.187-203

Vrijens, B.; Goetghebeur, E.; de Klerk, E.; et al. (2005). Modelling the association between adherence and viral load in HIV-infected patients. Statistics in Medicine, Vol.24, pp.2719-2731

Wakefield, J. C. (1996). The Bayesian approach to population Pharmacokinetic models. Journal of the American Statistical Association, Vol.91, pp.61-76

Wu, H. \& Ding, A. A. (1999). Population HIV-1 dynamics in vivo: applicable models and inferential tools for virological data from AIDS clinical trials. Biometrics, Vol.55, pp.410-418

Wu, H.; Kuritzkes, D. R.; McClernon, D. R.; et al. (1999). Characterization of viral dynamics in Human Immunodeficiency Virus Type 1-infected patients treated with combination antiretroviral therapy: relationships to host factors, cellular restoration and virological endpoints. Journal of Infectious Diseases, Vol.179, pp.799-807

Wu, H.; Huang, Y.; Acosta E. P.; et al. (2005). Modeling long-term HIV dynamics and antiretroviral response: effects of drug potency, pharmacokinetics, adherence and drug resistance. J Acquir Immune Defic Syndr., Vol.39, pp.272-283 


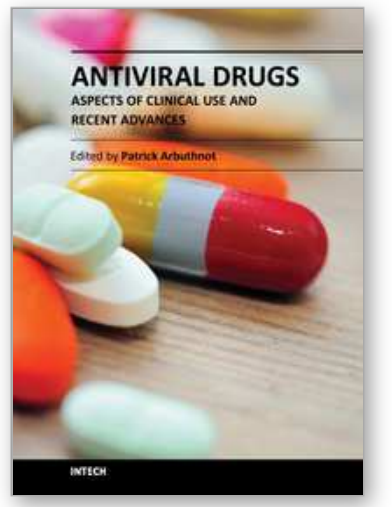

\author{
Antiviral Drugs - Aspects of Clinical Use and Recent Advances \\ Edited by Dr. Patrick Arbuthnot
}

ISBN 978-953-51-0256-4

Hard cover, 194 pages

Publisher InTech

Published online 14, March, 2012

Published in print edition March, 2012

The articles that appear in Antiviral Drugs - Aspects of Clinical Use and Recent Advances cover several topics that reflect the varied mechanisms of viral disease pathogenesis and treatment. Clinical management and new developments in the treatment of virus-related diseases are the two main sections of the book. The first part reviews the treatment of hepatitis $C$ virus infection, the management of virus-related acute retinal necrosis, the use of leflunomide therapy in renal transplant patients, and mathematical modeling of HIV-1 treatment responses. Basic research topics are dealt with in the second half of the book. New developments in the treatment of the influenza virus, the use of animal models for HIV-1 drug development, the use of single chain camelid antibodies against negative strand RNA viruses, countering norovirus infection, and the use of plant extracts to treat herpes simplex virus infection are described. The content of the book is not intended to be comprehensive, but aims to provide the reader with insights into selected aspects of established and new viral therapies.

\title{
How to reference
}

In order to correctly reference this scholarly work, feel free to copy and paste the following:

Yangxin Huang (2012). Modeling Virologic Response in HIV-1 Infected Patients to Assess Medication Adherence, Antiviral Drugs - Aspects of Clinical Use and Recent Advances, Dr. Patrick Arbuthnot (Ed.), ISBN: 978-953-51-0256-4, InTech, Available from: http://www.intechopen.com/books/antiviral-drugs-aspects-ofclinical-use-and-recent-advances/modeling-virologic-response-in-hiv-1-infected-patients-to-assess-medicationadherence

\section{INTECH}

open science | open minds

\section{InTech Europe}

University Campus STeP Ri

Slavka Krautzeka 83/A

51000 Rijeka, Croatia

Phone: +385 (51) 770447

Fax: +385 (51) 686166

www.intechopen.com

\section{InTech China}

Unit 405, Office Block, Hotel Equatorial Shanghai

No.65, Yan An Road (West), Shanghai, 200040, China 中国上海市延安西路65号上海国际贵都大饭店办公楼 405 单元

Phone: +86-21-62489820

Fax: +86-21-62489821 
(C) 2012 The Author(s). Licensee IntechOpen. This is an open access article distributed under the terms of the Creative Commons Attribution 3.0 License, which permits unrestricted use, distribution, and reproduction in any medium, provided the original work is properly cited. 